\section{ICSI: Spermien aus Hoden oder Ejakulat?}

\author{
Wenn Spermien aus dem Ejakulat für eine intrazytoplasmatische Spermien- \\ injektion (ICSI) nicht verfügbar sind, kann die Entnahme von Spermien aus \\ den Testes möglicherweise eine Option sein. Hinweise dafür liefert eine \\ retrospektive Studie aus China.
}

—ür die Studie werteten Dr. Xianfeng - Cui et al. die Befunde von fast 300 Männern mit Kryptozoospermie aus. Bei 214 Männern wurden für die ICSI Spermien aus dem Ejakulat verwendet, bei 71 Spermien, die durch testikuläre Spermienaspiration (TESA) oder konventionelle testikuläre Spermienextraktion (TESE) gewonnen worden waren. Im Fokus der Untersuchung standen Embryoqualität, Befruchtungserfolg, Implantation, Schwangerschaft und Geburtenrate nach einer ICSI.

Die Fertilisationsrate per ICSI lag bei Verwendung von Spermatozoen aus dem Ejakulat bei $59,6 \%$, in der Vergleichsgruppe bei $60,6 \%$. Der Anteil der so erzeugten Embryonen mit guter Qualität betrug dagegen $36,8 \%$ versus $46,1 \%$, die
Implantationsrate $30,7 \%$ versus $52,1 \%$. Die Schwangerschaftsrate lag bei 33,3\% versus 53,6 \% und die Geburtenrate bei $27,1 \%$ versus 44,6\%. Damit scheinen die Erfolgsraten bei Implantation, Schwangerschaft und Geburt mit aus den Hoden gewonnenen Spermatozoen signifikant höher.

Fazit: Nach Ansicht von Cui et al. sind direkt aus den Hoden gewonnene Spermatozoen nicht den schädlichen Einflüssen des Urogenitaltrakts oder von freien Sauerstoffradikalen ausgesetzt. Es gebe dadurch seltener DNA-Schäden und eher Embryonen mit guter Qualität. Die Männer, bei denen am Tag der Oozytenentnahme eine TESA oder TESE angewendet wurde, hatten im Ejakulat keine lebensfähigen Spermatozoen, obwohl das im Zeitraum davor der Fall war. Der Nachteil der Spermatozoengewinnung per TESE/TESA ist die erhöhte Rate an Komplikationen durch Blutungen oder Infektionen und an testikulärer Atrophie. Die Ärzte geben zudem zu bedenken, dass keine „Intention-to-treat“ Analyse vorgenommen wurde, denn von den 302 Männern mit Kryptozoospermie wurden nur 285 in der Endauswertung berücksichtigt. Einschränkend weisen sie zudem darauf hin, dass die Komplikationsrate nach TESE/TESA bei $20 \%$ gelegen habe und sich bei einigen Patienten mit dieser Methode für die Invitro-Fertilisation keine Spermien gewinnen ließen. Schließlich seien die Spermatozoen nicht auf mögliche Defekte untersucht worden.

Peter Leiner

Cui $X$ et al. Comparison of the clinical outcomes of intracytoplasmic sperm injection between spermatozoa retrieved from testicular biopsy and from ejaculate in cryptozoospermia patients. Urology 2016; doi: 10.1016/j.urology.2016.08.071.

\title{
Erörterung der Lebenserwartung und verbessertes Krankheitsverstehen
}

\begin{abstract}
Erst das genaue Verstehen der eigenen Erkrankung befähigt den Patienten, informierte Entscheidungen zu treffen,zum Beispiel für eine palliative Versorgung. Nun wurde in einer Studie geprüft, ob Gespräche von Arzt und Patient über die Prognose tatsächlich das korrekte Krankheitsverstehen fördern.
\end{abstract}

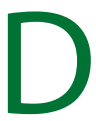
azu wurden 178 Patienten mit fortgeschrittenen Tumoren interviewt, die gegenüber der vorausgegangenen Chemotherapie refraktär waren und bei denen die Onkologen die mittlere Lebenserwartung auf bis zu sechs Monate schätzten. Die Patienten wurden vor und nach Besprechung der Ergebnisse einer Re-Staging-Bildgebung befragt. Ihr Krankheitsverständnis wurde anhand von vier Variablen bestimmt: der Erkenntnis, dass eine terminale Krankheitsphase erreicht war, dass die Erkrankung nicht mehr heilbar war und ein fortgeschrittenes Stadium vorlag sowie der Einsicht, dass die Lebenserwartung eher Monate als Jahre betrug. Vor der Konsultation mit einer Besprechung des Re-Staging-Scans zeigten nur 9 (5\%) der 178 Patienten ein vollständig korrektes Verständnis ihrer Erkrankung, das heißt nur diese neun Patienten beantworteten alle vier Fragen zur prognostischen Einschätzung korrekt. Nach der Restaging-Konsultation berichteten 18 Patienten $(10 \%)$ lediglich über das aktuelle Gespräch mit ihrem Onkologen zu diesem Thema; 24 (13\%) gaben sowohl das aktuelle als auch frühere Gespräche an; 68 (38\%) wiederum erinnerten sich dagegen nur an frühere Erörterungen. Überraschenderweise berichteten $68 \mathrm{~Pa}$ tienten $(38 \%)$, dass sie sich noch niemals mit ihrem Onkologen über Prognose und Lebenserwartung unterhalten hätten. Bezüglich der korrekten Einschät- zung ihrer Lage zeigten vor allem die $\mathrm{Pa}$ tienten signifikante Verbesserungen im Krankheitsverstehen, die über ein aktuelles Gespräch zu diesem Thema berichtet hatten. In dieser Subgruppe ergab sich eine mittlere Zunahme des Verstehens-Scores um 0,62 ( $p=0,002)$, und in der Gruppe mit aktueller und früherer Erörterung um $0,37(p=0,028)$. In den beiden anderen Gruppen fand sich keine Zunahme der Verstehens-Scores.

Fazit: Patienten mit fortgeschrittenem Tumorleiden, die sich an eine kürzliche Besprechung der infausten Prognose und der begrenzten Lebenserwartung mit ihrem Onkologen erinnerten, zeigten ein besseres Verständnis für die terminale Natur ihrer Erkrankung.

Brigitte Schalhorn

Epstein AS et al. Discussions of life expectancy and changes in illness understanding in patients with advanced cancer. J Clin Oncol. 2016; 34(20): 2398-403. 